\title{
Research summer camp in photonics
}

Elizaveta Buyanovskaya, Maksim Melnik, Vladimir Egorov, Artur Gleim, Svetlana Lukishova, et al.

Elizaveta Buyanovskaya, Maksim Melnik, Vladimir Egorov, Artur Gleim, Svetlana Lukishova, Sergei Kozlov, Xi-Cheng Zhang, "Research summer camp in photonics," Proc. SPIE 10452, 14th Conference on Education and Training in Optics and Photonics: ETOP 2017, 104523H (16 August 2017); doi: $10.1117 / 12.2266536$

SPIE Event: 14th Conference on Education and Training in Optics and Photonics, ETOP 2017, 2017, Hangzhou, China 


\title{
Research summer camp in photonics
}

\author{
Elizaveta Buyanovskaya ${ }^{\mathrm{a}}$, Maksim Melnik ${ }^{\mathrm{a}}$, Vladimir Egorov ${ }^{\mathrm{a}}$, Artur Gleim ${ }^{\mathrm{a}}$, Svetlana \\ Lukishova $^{\mathrm{b}}$, Sergei Kozlov ${ }^{\mathrm{a}}$, and Xi-Cheng Zhang ${ }^{\mathrm{a}, \mathrm{b}}$ \\ aTMO University, 197101, Kronverksky, 49, Saint-Petersburg, Russia \\ ${ }^{\text {b}}$ The Institute of Optics, University of Rochester, 275 Hutchison Rd, Rochester, NY 14620, \\ USA
}

\begin{abstract}
ITMO University and the University of Rochester became close partners several years ago. One of the first outcomes of this mutually beneficial partnership was the creation of International Institute of Photonics and Optical Information Technologies led by Prof. Sergei Kozlov and Prof. Xi-Cheng Zhang. Universities have created a double Masters-degree program in optics in 2014, and several ITMO students have been awarded degrees from Rochester. At the same time ITMO University organizes Summer Research camp in Photonics for University of Rochester students. Students spent two weeks in the Northern Capital of Russia learning about the emerging practical applications of femtosecond optics, terahertz biomedicine and quantum information technologies.
\end{abstract}

Keywords: short term educational program, quantum cryptography, femtosecond optics and technologies, terahertz technologies

\section{INTRODUCTION}

Scientific collaboration between ITMO University and the University of Rochester involves three main fields of research: femtosecond optics and technologies, quantum information technologies, and terahertz biomedicine. Three years ago an International Education Program was launched as well, which allowed promising Russian students to take one year in Rochester during Coursework MS program in Optical Design and Engineering. Five students already became owners of MS degrees diplomas of both universities. At the same time, the program opened the doors to American students for visiting Saint Petersburg and participating in real-life experimental research activities. Undergraduate students from the Institute of Optics travel to Russia for summer practice at the Research Summer camp in Photonics, giving them a unique opportunity to take part in cutting edge scientific research and gain additional experience in laboratory work. ${ }^{1}$ For the last three years, ITMO University welcomed nineteen Rochester students. The research directions covered all areas of science the two universities are cooperating in.

\section{SUMMER PHOTONICS CAMP PROGRAM}

Students spent two weeks in St.Petersburg learning about the emerging practical applications of femtosecond optics, terahertz biomedicine and quantum information technologies. Each student worked with one of the scientific laboratories of the International Institute Photonics and Optoinformatics of ITMO University. They not only worked in teams but also carried out their own research that contributed to the overall work of the lab. Research focused on three areas: femtosecond optics and femtotechnology, terahertz medicine, and quantum science. Together with their ITMO fellows, some of the guests were involved in quantum communications project and shared a unique experience of working on the first Russian metropolitan quantum network.

Last year, Huiyan Li, a first year bachelor, worked in Femtosecond optics and technology laboratory, where she studied nonlinear properties of photochromic glass used for eye protection from intensive optical radiation. The research was performed with state-of-the-art femtosecond pump-probe spectroscopy instrumentation that

Further author information: (Send correspondence to Elizaveta Buyanovskaya)

Elizaveta Buyanovskaya: E-mail: lee.buyanovskaya@niuitmo.ru, Telephone: +79219712302

14th Conference on Education and Training in Optics and Photonics: ETOP 2017, edited by Xu Liu,

Xi-Cheng Zhang, Proc. of SPIE Vol. 10452, 104523H · @ 2017 ICO, IEEE, OSA, SPIE

CCC code: $0277-786 \mathrm{X} / 17 / \$ 18 \cdot$ doi: $10.1117 / 12.2266536$

Proc. of SPIE Vol. $10452104523 \mathrm{H}-1$ 


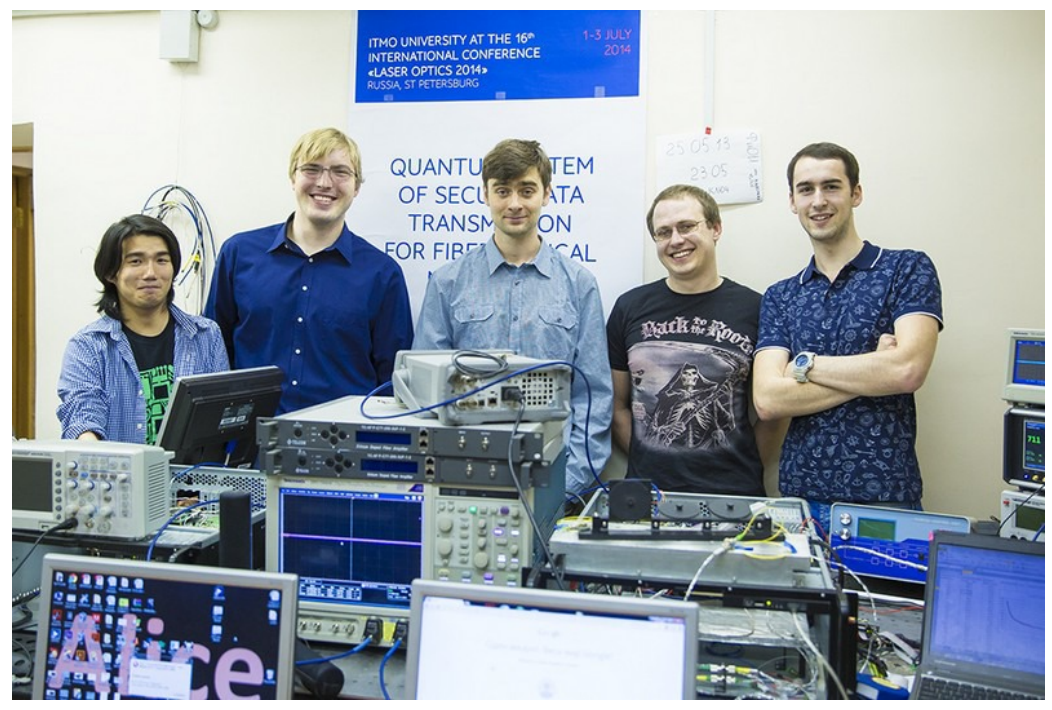

Figure 1. University of Rochester students involved in quantum communication project in 2015

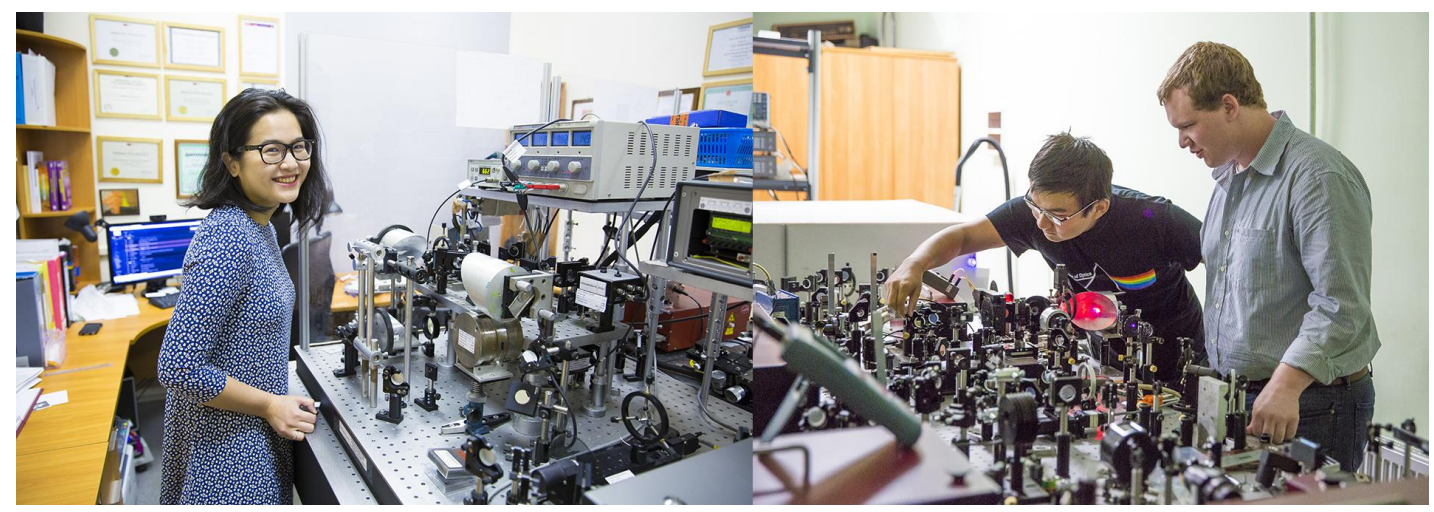

Figure 2. University of Rochester students involved in femtosecond and terahertz projects in 2015

used supercontinuum for pumping. ${ }^{2}$ Three students were involved in studying digital holography images. Li Guoxin compared two algorithms for reconstructing off-axis digital holograms used in image plane configuration. Liu Weidi participated in a project dedicated to improving the Terahertz Pulse Time-Domain Holography ( $\mathrm{THz}$ PTDH) method that was proved perspective for phase imaging in the terahertz frequency range. ${ }^{3}$ The work done by Weidi will allow increasing measurement speed by several times. Xia Zhenhi chose wavefront adaptive optimization through scattering media as her topic. She participated in creating a device for focusing optical radiation in highly scattering media at centimeter depth. This approach is very perspective for many biomedical applications, such as photodynamic therapy. Other research branches included investigation of optical properties of artificial media, simulation of optical devices on basis of transformation optics and studying optical properties of bio-tissues and cells, which were covered by Ma Shiyu, Song Wanyue and Yu Lu. Two of the nine guests, Eric Herbert and Barry Magenya, picked a project in the field of quantum information technologies. ${ }^{4}$ Eric investigated the properties of perspective single-photon sources, while Barry focused on technological features of high-speed quantum communication systems. Most of the time, they were working in pair and carried out all experiments together. At the end of the course, the students presented their projects before scientific staff of the department and answered the questions.

In 2016, an icing on the cake was educational practice on the first Russian quantum network. Launched in 2014, it connects two buildings of ITMO University through standard telecommunication fibers. Quantum communication networks offer unique possibilities for users to exchange information through optical links that 


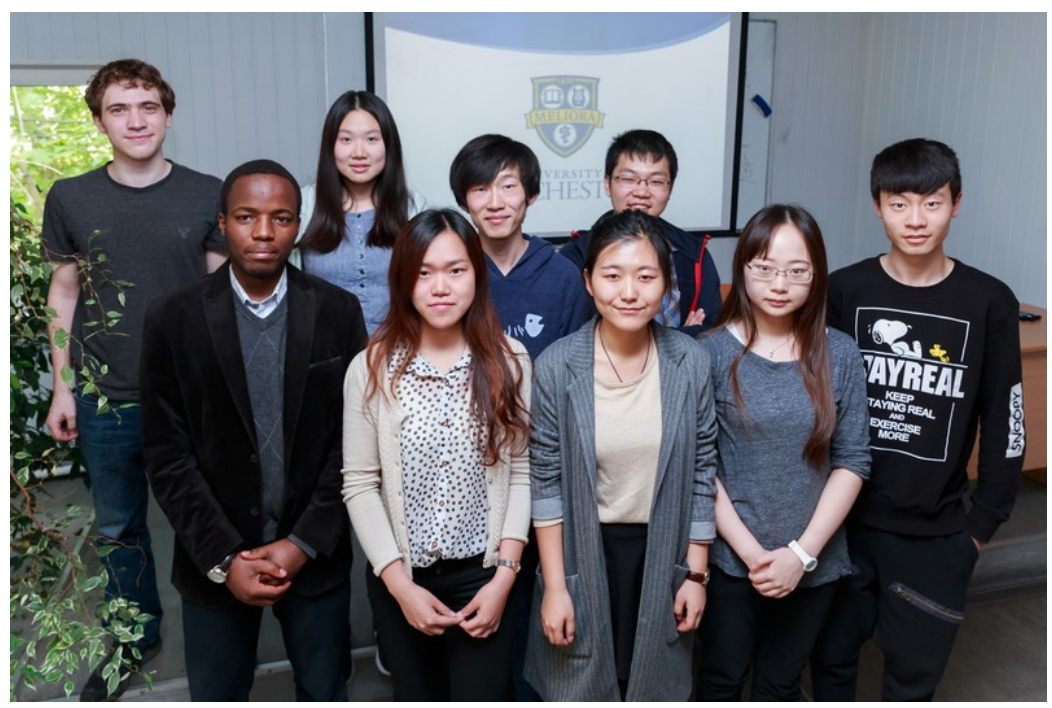

Figure 3. Participants of 2016 Summer camp in Photonics

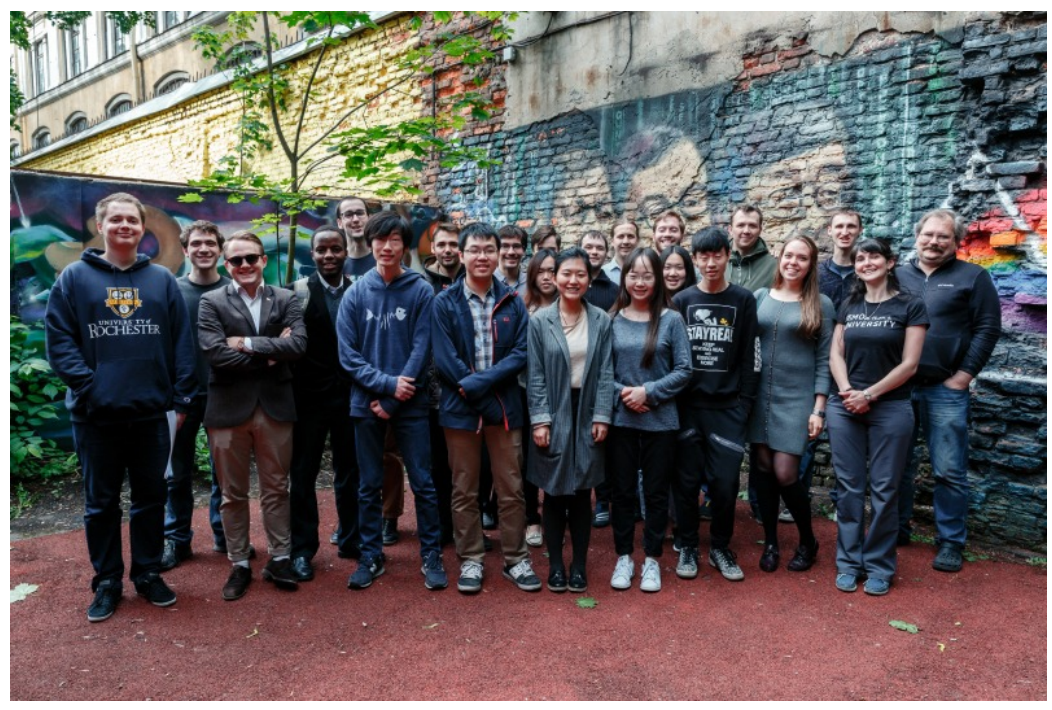

Figure 4. Participants of 2016 Summer camp in Photonics together with ITMO researches

cannot be eavesdropped thanks to the laws of quantum physics that do not allow quantum signal carriers single photons to be split or copied. Prior to becoming quantum network operators, the students learned features of quantum key distribution during lectures and participated in seminars about several closely related topics, such as quantum optical instrumentation, which allowed then to understand the setup, successfully perform measurements, and finally exchange the encrypted messages that contained the logos of both universities. The measured sifted key rate reached $1 \mathrm{Mbit} / \mathrm{s}$ on a $1.6 \mathrm{~dB}$ line connecting two ITMO buildings through a standard underground optical cable. Both Barry and Eric were thrilled to take part in this event, since there are currently only a few quantum networks in world, most of them not used for education.

In 2017 ITMO University is getting ready to welcome four students. This year the summer camp will include extensive lecture program.

ITMO University is proud to provide a unique possibility for the U.S. students to learn something new and acquire outstanding skills, and hopes that their collaboration with University of Rochester will continue to thrive. 


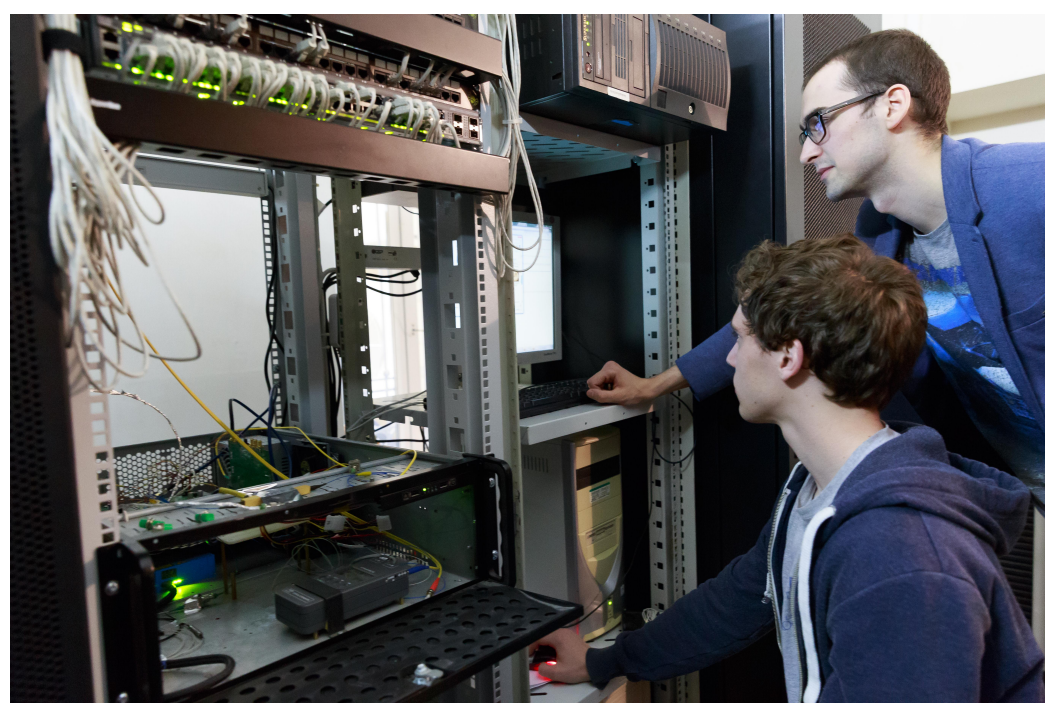

Figure 5. Educational practice on the first Russian quantum network

\section{ACKNOWLEDGMENTS}

The authors would like to thank Daniel Smith, Undergraduate Program Manager, The Institute of Optics, Dr. Nikolay Petrov, leading researcher, ITMO University, Dr. Anton Tsypkin, head of femtosecond optics and femtotechologies laboratory, ITMO University, Dr. Mikhail Khodzitsky, head of terahertz biomedicine laboratory, ITMO University, Dr. Anna Vozianova, ITMO University Dr. Olga Smolyanskaya, ITMO University.

This work was supported by Government of Russian Federation, Grant 074-U01.

\section{REFERENCES}

[1] Lukishova, S. G., Kozlov, S. A., and Zavestovskaya, I. N., "A Rochester-Russia Collaboration." Optics and Photonics News, November 2016 http://www.osa-opn.org/home/articles/volume_27/november_2016/ departments/a_rochester-russia_collaboration/.

[2] Tcypkin, A. N., Putilin, S. E., Melnik, M. V., Makarov, E. A., Bespalov, V. G., and Kozlov, S. A., "Generation of high-intensity spectral supercontinuum of more than two octaves in a water jet," Appl. Opt. 55, 8390-8394 (Oct 2016).

[3] Belashov, A. V., Zhikhoreva, A. A., Belyaeva, T. N., Kornilova, E. S., Petrov, N. V., Salova, A. V., Semenova, I. V., and Vasyutinskii, O. S., "Digital holographic microscopy in label-free analysis of cultured cells' response to photodynamic treatment," Opt. Lett. 41, 5035-5038 (Nov 2016).

[4] Gleim, A. V., Egorov, V. I., Nazarov, Y. V., Smirnov, S. V., Chistyakov, V. V., Bannik, O. I., Anisimov, A. A., Kynev, S. M., Ivanova, A. E., Collins, R. J., Kozlov, S. A., and Buller, G. S., "Secure polarizationindependent subcarrier quantum key distribution in optical fiber channel using bb84 protocol with a strong reference," Opt. Express 24, 2619-2633 (Feb 2016). 